Ann. Biol. anim. Bioch. Biophys., 1977, 17 (1), 9-19.

\title{
Motricité utérine de la Brebis, avant, pendant et après la parturition spontanée ou après traitement par la dexaméthasone
}

\author{
par Marie-Jeanne PRUD'HOMME, M. J. BOSC
}

avec la collaboration technique de Agnès NICOLLE, Y. LEBRETON et A. LOCATELLI

Station de Physiologie de la Reproduction, I. N. R. A., Nouzilly, 37380 Monnaie (France)

Summary. Uterine activity in ewe before, during and after spontaneous parturition or after dexamethasone priming.

Uterine activity is compared in ewe having spontaneous parturition and in dexamethasone-primed ewe. This comparison is made from in vivo recording of myometrium electromyographic activity (EMG) and of mechanical activity determined by variations in intra-uterine pressure.

Results show that dexamethasone treatment does not alter the development of EMG myometrium activity in the preparturient ewe. Whether before spontaneous or induced birth, 3 successive phases are observed : the first ends between 24 and 20 hours before lambing ; it is characterized by $250-300$ second bursts of EMG activity four to five times per hour. After a transitory phase of several hours, EMG activity during the last 16-17 hours is characterized by $40-50$ second bursts synchronous with variations in intra-uterine pressure.

$U_{p}$ to birth, bursts are increasingly regular and frequent while the amplitude of variations in intra-uterine pressure increases.

\section{Introduction.}

Chez la Brebis, il est établi que la fonction hypophyso surrénalienne du foetus détermine et contrôle le mécanisme de la naissance (Liggins ef al., 1967 ; Drosł et Holm, 1968 ; Liggins, 1968). Ainsi, la perfusion du fœfus par un corticostéroïde de synthèse (la dexaméthasone) est suivie de la mise-bas prématurée (Liggins et al., 1973 ; 
Currie et al., 1973) ef l'administration du même produit à la mère peut induire l'agnelage (Bosc, 1970). Dans le premier cas, on suppose que ce corticostéroïde agit comme le ferait le cortisol foetal, en relation avec les œstrogènes; dans le deuxième cas, il agirait par rétroaction négative au niveau hypophysaire foetal (Bosc, 1972 ; Bosc et Fèvre, 1974, 1975). Dans ces deux cas, on n'attribue pas aux corticostéroïdes (cortisol ou dexaméthasone) un effet direct de stimulation sur l'activité utérine. Cependant, Mossman et Conrad (1969), in vitro, et Zasztowt et Wisniewski (1973), in vivo, ont montré que la prednisolone ou le cortisol stimulent l'activité du myomètre chez la Femme. Afin d'éprouver cette possibilité chez la Brebis, nous avons enregistré l'activité électromyographique (EMG) du myomètre avant, pendant et après la parturition spontanée et nous l'avons comparée à celle d'animaux qui ont été traités par la dexaméthasone.

\section{Technique.}

Sept brebis gravides de race lle-de-France sont utilisées. L'activité EMG de l'utérus est recueillie sur l'animal éveillé à l'aide d'électrodes de platine bipolaires selon la technique de Rousseau et Prud'homme (1974). Ces électrodes sont fixées aux 2 extrémités de la corne gravide de l'utérus lors d'une laparotomie basse pratiquée sous anesthésie générale (fluothane après induction par un mélange de penthotal et de nembutal) vers le $130^{\circ}$ jour de la gestation (du $129 \mathrm{e}$ au $132^{\mathrm{e}}$ ). Les variations de la pression intra-utérine sont détectées par l'extrémité libre d'un catheter rempli de solution physiologique, mis en place dans la poche allantoïdienne; elles sont transformées en variations de potentiel par un transducteur de type inductif et visualisées sur papier en même temps que les activités électromyographiques du myomètre, grâce à un inscripteur à plumes classique (polygraphe E.C.E.M. utilisé à une vitesse de $1,5 \mathrm{~cm} / \mathrm{s}$ ).

Les enregistrements débutent $48 \mathrm{~h}$ après l'intervention chirurgicale, chaque brebis étant placée dans une cage ; ils sont poursuivis jusqu'à l'expulsion des agneaux. Dans ces conditions, 4 mise-bas spontanées se sont produites précocement entre le $137^{\mathrm{e}}$ ef le $143^{\mathrm{e}}$ jour de la gestation car dans les conditions habituelles d'élevage, la plupart des brebis agnellent entre le $144^{\mathrm{e}}$ et le $151^{\mathrm{e}}$ jour de la gestation. Compte tenu de cette observation, l'administration de dexaméthanose a été avancée chez les 3 brebis traitées; deux animaux ont reçu une seule injection intra-musculaire de $16 \mathrm{mg}$ de ce produit le $136^{\mathrm{e}}$ et le $137^{\mathrm{e}}$ jour de la gestation et ont agnelé respectivement $31 \mathrm{~h}$ et $44 \mathrm{~h}$ après, ce qui correspond aux délais habituellement observés après ce traitement (Bosc ef Fèvre, 1974). La $3^{\mathrm{e}}$ brebis a reçu 2 injections, l'une le $138^{\mathrm{e}}$ jour, l'autre le $142^{\mathrm{e}}$ jour ; l'agneau a été extrait par césarienne en raison d'une dystocie de présentation, $4 \mathrm{~h}$ cprès la rupture de la poche des eaux (soit $37 \mathrm{~h}$ après la seconde injection). Tous les agneaux sont nés vivants à l'exception de ce dernier.

Chez 2 brebis ( 1 témoin et 1 traitée), les enregistrements sont poursuivis au cours de la période post-partum (environ $30 \mathrm{~h}$ ).

Pour l'analyse, nous avons caractérisé, à différents moments par rapport à la parturition, l'activité EMG de l'utérus par la durée et la fréquence des périodes d'activité. De plus, l'évolution du temps total d'activité par heure a été suivie pour chaque animal. 


\section{Résultats.}

\section{A) Motricité utérine jusqu'à la mise-bas}

\section{Mise-bas spontanée.}

Au cours de la dernière semaine de la gestation, l'évolution de l'activité EMG permet de définir trois phases caractéristiques

La première phase se termine environ $24 \mathrm{~h}$ avant le part; au cours de celle-ci, l'activité EMG se manifeste 4 à 5 fois par heure, par périodes de 250 à $300 \mathrm{~s}$, constituées de potentiels d'action dont l'amplitude est de $200 \mu \mathrm{V}$ environ et dont la fréquence moyenne est de 1,1 à 1,2 potentiel par seconde. Ces périodes actives sont séparées par
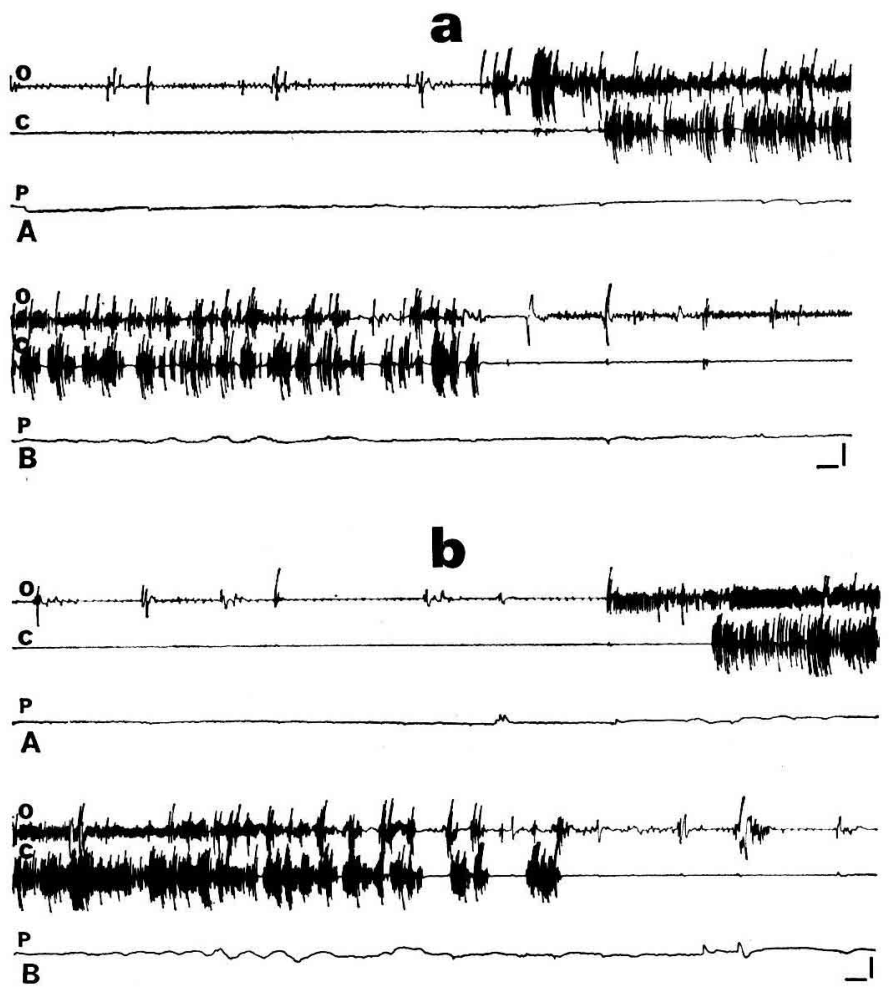

FIG. 1. - Activité EMG de l'utérus chez la brebis

a) $1 \mathrm{~h}$ avant, b) $1 \mathrm{~h}$ après une injection de dexaméthasone $(16 \mathrm{mg}), 44 \mathrm{~h}$ avant le part.

(O) partie ovarienne de l'utérus, (C) partie cervicale de l'utérus, $(P)$ pression intra-allantoïdienne.

(Les lignes $\mathrm{O}, \mathrm{C}$ ef $\mathrm{P}$ des tracés $\mathrm{A}$ et $\mathrm{B}$ se font suite sans recouvrement.)

Etalonnage : - amplitude : $400 \mu \mathrm{V}(\mathrm{O}$ ef $\mathrm{C}), 10 \mathrm{~mm} \mathrm{Hg}(\mathrm{P})$ - temps : $10 \mathrm{~s}$. 
des silences électriques de 400 à $500 \mathrm{~s}$ (tabl.). Les variations de la pression intrautérine sont faibles $(3-5 \mathrm{~mm}$ de $\mathrm{Hg})$. La figure $1 a$ illustre ce premier type d'activité au cours d'un enregistrement effectué $45 \mathrm{~h}$ avant le part. Le temps total d'activité mesuré par heure $(1000$ à $1500 \mathrm{~s})$ de même que la durée et la fréquence des périodes qui la composent, restent, pour chaque brebis, sensiblement constants (fig. 2 et tabl.).

La deuxième phase peut être définie comme une phase de transition qui se termine 16-17 h environ avant le part. En effet, des bouffées d'activité brèves ( 30 à $70 \mathrm{~s}$ ) apparaissent entre des bouffées d'activité plus longues (100 à $300 \mathrm{~s}$ ). La durée de ces dernières diminue peu à peu (tabl. et fig. 4a). A ce moment l'amplitude des variations de la pression intra-utérine reste faible.

La troisième phase se termine par l'expulsion de l'agneau. Elle est caractérisée par des salves de $45 \mathrm{~s}$ en moyenne ( 40 à $80 \mathrm{~s}$ ) qui apparaissent de plus en plus régulièrement et qui sont synchrones des variations de la pression intra-utérine (tabl. et fig. 5a). La fréquence des potentiels au sein de ces salves augmente également et

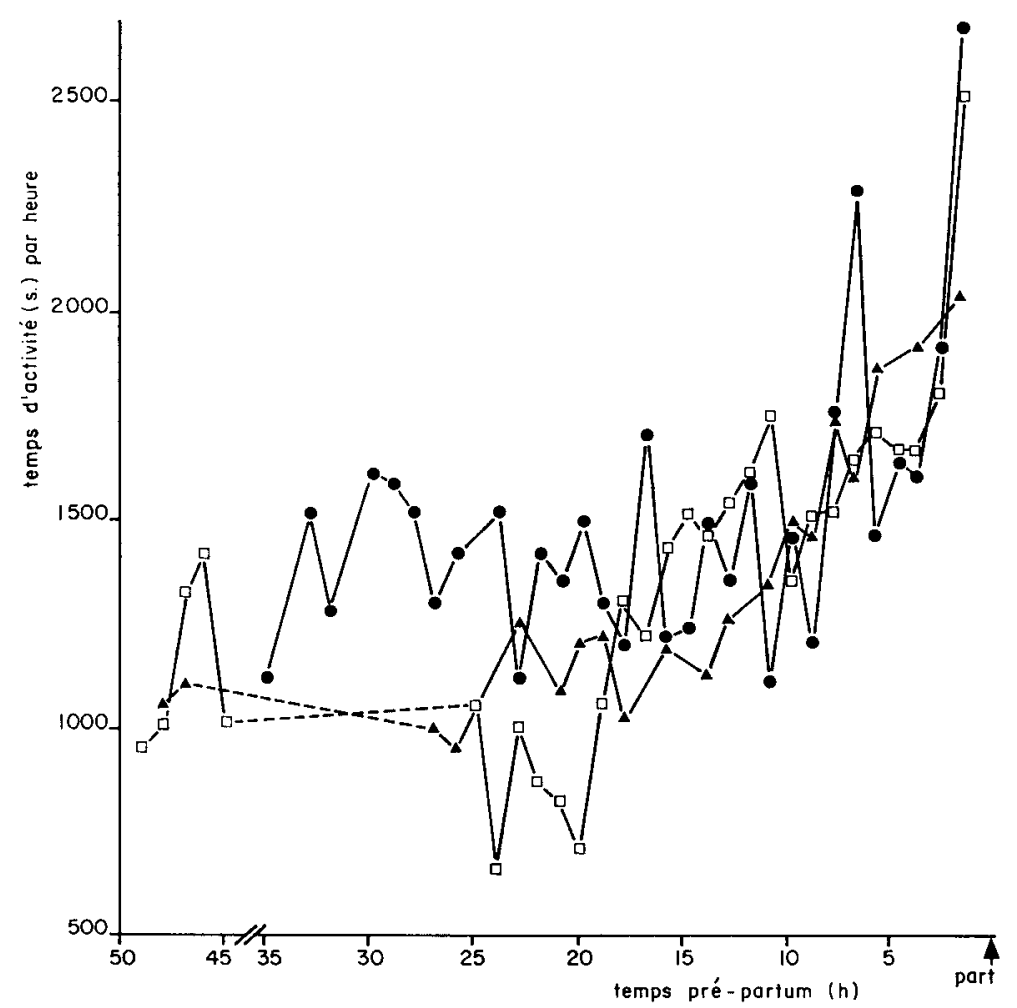

FIG. 2. - Evolution du femps d'activité de l'utérus (mesuré en secondes par heure) ou cours des trois derniers jours de la gestation, chez 3 brebis, dont la mise-bas est spontanée. Le temps d'activité par heure varie peu, jusqu'à 15 h environ avant le part ; il double ensuite au cours des dernières heures de gestation. 
atteint 1,7 à 2,2 potentiels par seconde ; leur amplitude atteint 400 à $600 \mu \mathrm{V}$ au moment de l'expulsion. La fréquence des salves augmente tout au long de cette troisième phase jusqu'à la mise-bas (tabl.). Il en résulte que le temps total d'activité mesuré par heure s'accroît lui aussi jusqu'au part (fig. 2).

Signalons en outre que, au cours de la dernière semaine de la gestation, l'activité EMG apparaît en premier indifféremment au niveau des électrodes placées à l'une ou l'autre extrémité de la corne gravide. C'est ce que l'on observe par exemple sur la figure $5 a$.

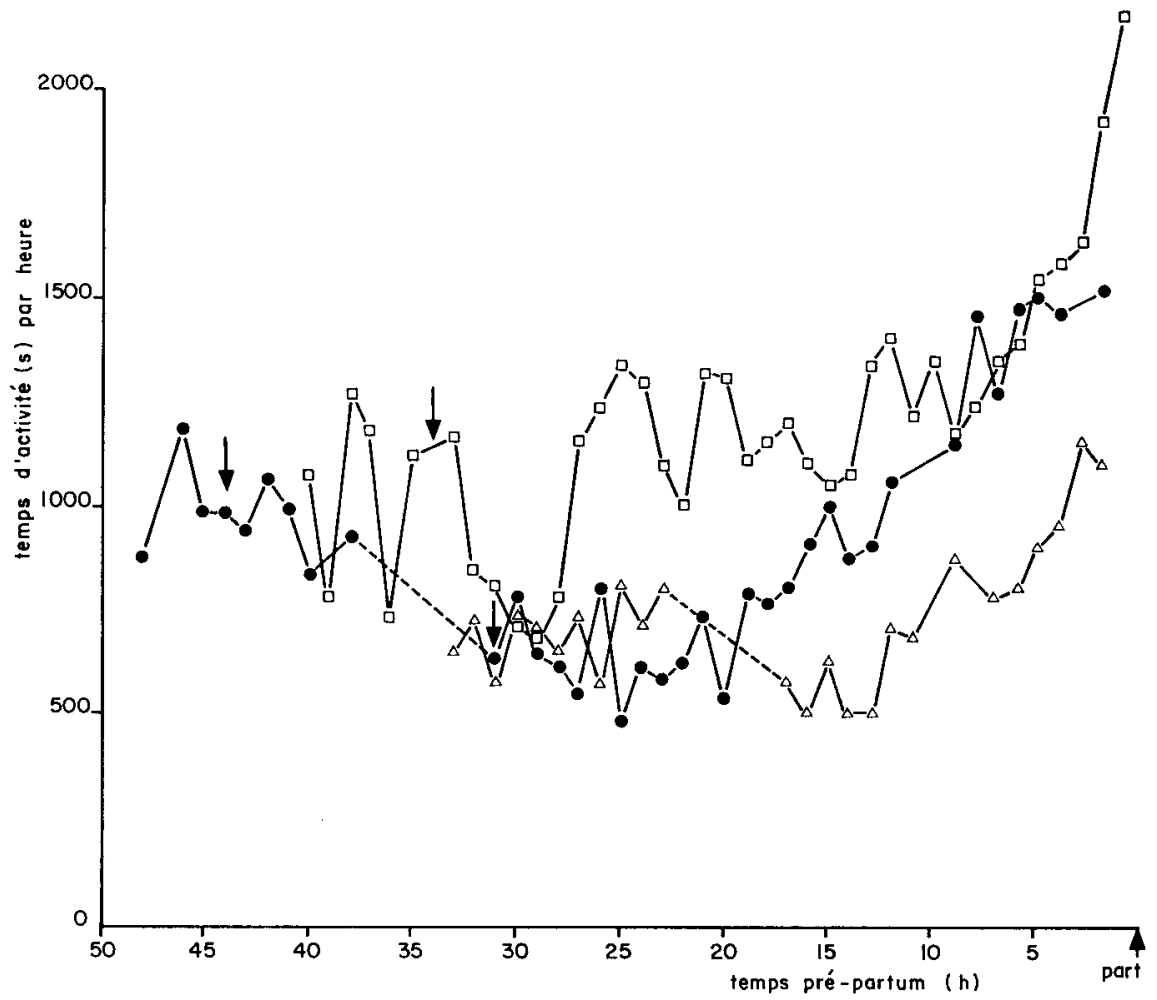

FIG. 3. - Evolution du temps d'activité de l'utérus chez 3 brebis dont la mise-bas est induite par la dexaméthasone.

Injection de dexaméthasone $\downarrow$. L'évolution du temps d'activité par heure est comparable à celle des brebis dont la mise-bas est spontanée.

2. Mise-bas induite par le dexaméthasone.

La motricité utérine dans ces conditions suit une évolution analogue à celle qui est observée lors de la mise-bas spontanée. Dans nos conditions, l'administration de dexaméthasone a été faite entre 30 et $45 \mathrm{~h}$ avant l'expulsion de l'agneau, c'est-à-dire 
au cours de la première phase décrite précédemment. Dans les heures qui ont suivi cette administration, la fréquence ef la durée des périodes de l'activité EMG ne subissent aucune modification (fig. $1 b$ et tabl.). Comme chez les témoins, cette première phase se termine environ 24 h avant l'agnelage ef l'activité EMG se modifie alors au cours d'une phase transitoire (fig. $4 b$ ), qui se prolonge un peu plus longtemps (tabl.).
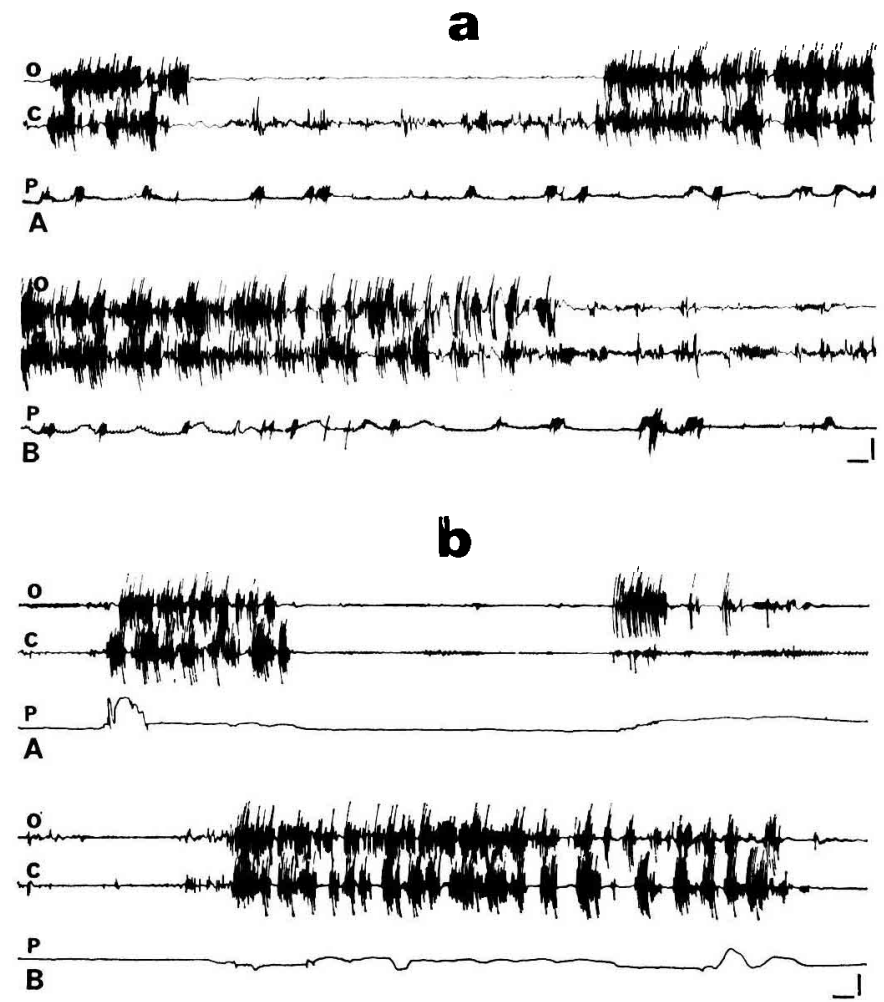

FIG. 4. - Activité EMG de l'utérus chez la Brebis $19 \mathrm{~h}$ avant le part.

a) brebis non traitée, b) brebis traitée par la dexaméthasone (mêmes enregistrements que sur la fig. 1).

Il en résulte que le dernier type d'activité ne prend place que 12 à $13 \mathrm{~h}$ avant le part, à des femps variables par rapport à l'injection de dexaméthasone. La figure $5 b$ illustre ce troisième type d'activité. La fréquence et l'amplitude des potentiels d'action qui caractérisent l'activité EMG au cours de ces trois phases ne présentent aucune différence notable avec celles des témoins. Le temps total de l'activité utérine mesurée par heure varie de la même manière que chez les témoins (fig. 3). 

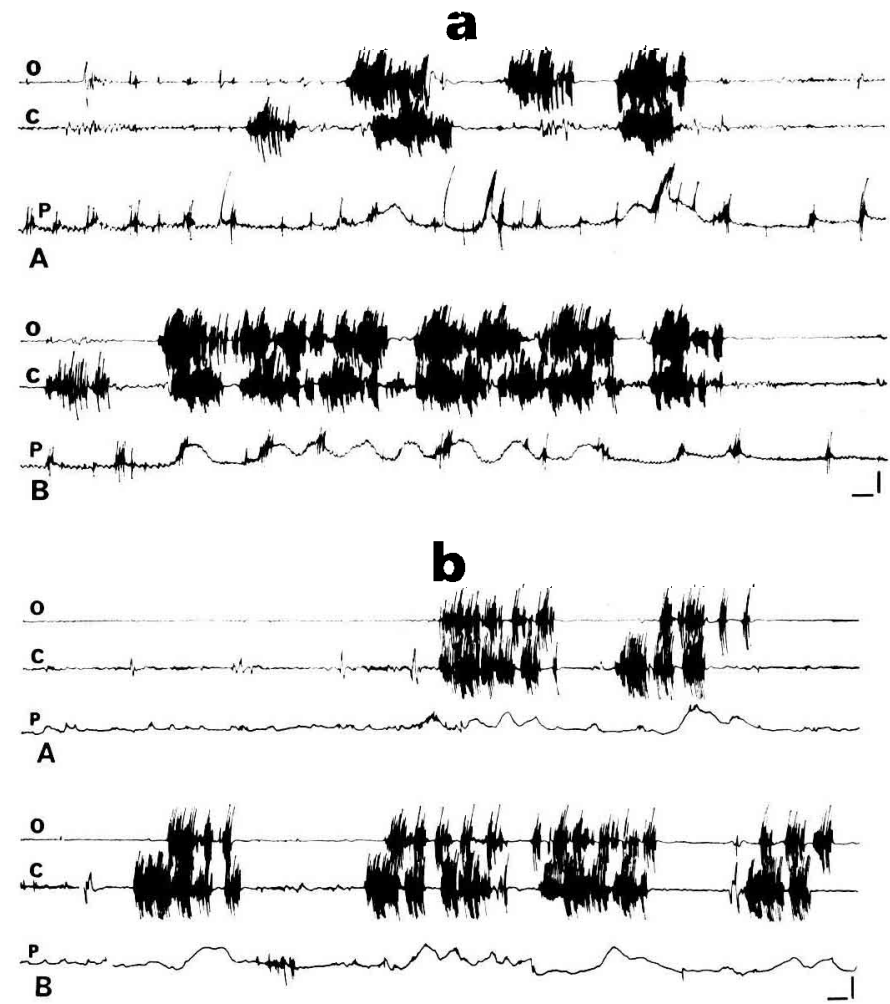

FIG. 5. - Activité EMG de l'utérus chez la brebis $10 \mathrm{~h}$ avant le part.

a) brebis non traitée, b) brebis traitée par la dexaméthasone (mêmes enregistrements que'sur la fig. 1).

\section{B) Motricité utérine après la mise-bas}

L'activité du myomètre est modifiée dès la fin de l'expulsion (fig. 6). Nous enregistrons dans le quart d'heure qui suit la mise-bas, au rythme de 0,8 par minute, des salves de $30 \mathrm{~s}$ constituées de potentiels dont l'amplitude a diminué (200 à $300 \mu \mathrm{V}$ ) (tabl.). Cette amplitude augmente ensuite avec le temps tandis que la fréquence des salves décroît régulièrement : $6 \mathrm{~h} 30$ après la mise-bas, nous ne comptons plus que 0,4 salve par minute. L'amplitude des potentiels est alors maximale (300 à $750 \mu \mathrm{V})$. A partir de $20 \mathrm{~h}$ post-partum, le rythme des salves est moins régulier : des périodes d'activité EMG de 10 à $20 \mathrm{mn}$ sont observées, séparées par des périodes silencieuses de 30 à $40 \mathrm{mn}$. Au bout de $30 \mathrm{~h}$, il ne subsiste plus que quelques potentiels d'action isolés de faible amplitude. 


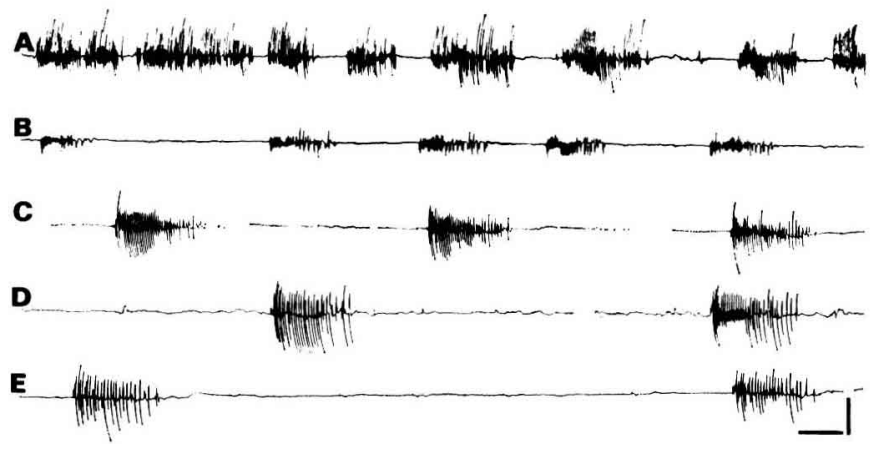

FIG. 6. - Activité EMG du myomètre chez la brebis

A : au cours du part ; $B: 10 \mathrm{mn}$ après le part ; $C: 6 \mathrm{~h} 30$ après le part;

$D: 12 \mathrm{~h}$ après le part; $E: 16 \mathrm{~h} 30$ après le part.

Noter après la mise-bas, la régularité du rythme des salves dont la fréquence décroît avec le temps. Ełalonnage : - amplitude : $500 \mu \mathrm{V}$ - temps : $20 \mathrm{~s}$.

TABLEAU

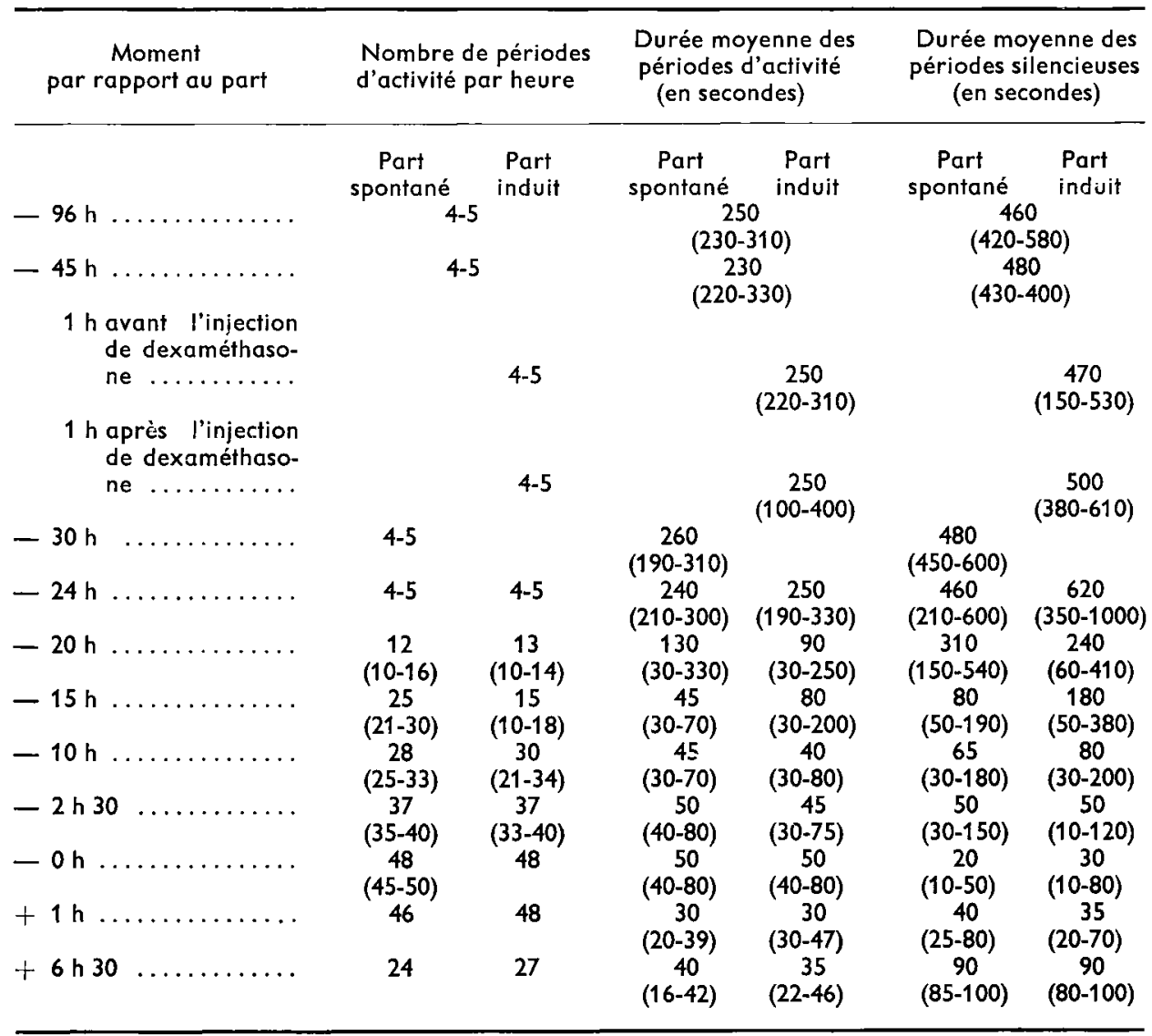




\section{Discussion.}

Comme l'indiquent ces résultats, la motricité utérine est stable au cours des derniers jours de la gestation. L'activité EMG ne se modifie qu'entre 24 et $20 \mathrm{~h}$ avant l'expulsion de l'agneau, ce qui correspond à ce qu'ont observé Naaktgeboren ef al. (1975). Cette modification est antérieure de quelques heures à celle des variations de la pression intra-utérine. En effet, celles-ci se développent, avec des amplitudes non négligeables pendant les 16 dernières heures de la gestation comme l'ont d'ailleurs décrit Hindson ef al. (1965) et Hindson et Ward (1973). A ce moment, l'allure par salves de l'activité EMG, contemporaine de l'activité mécanique traduit la mise en jeu synchrone et périodique d'un nombre de plus en plus grand de fibres musculaires. Ceci est vraisemblablement lié d'une part à la progestéronémie qui chute au cours des 2 derniers jours de la gestation (Bassett ef al., 1969) et d'autre part, au taux plasmatique de l'œstradiol $17 \beta$ qui s'élève brutalement au cours des $24 \mathrm{~h}$ qui précèdent la mise-bas (Challis et al., 1972 ; Thorburn ef al., 1972 ; Liggins ef al., 1973 ; Robertson et Smeaton, 1973).

L'activité EMG de l'utérus chez la brebis gravide avant la mise-bas n'est pas identique à celle observée chez la brebis en œstrus (Rousseau et Prud'homme, 1974). En effet, les salves de potentiels sont de plus longue durée (40 à $50 \mathrm{~s}$ ) dans le premier cas que dans le second (10 s), et elles apparaissent de façon moins régulière chez la brebis gravide. Comme l'expulsion de l'agneau est immédiatement suivie d'une plus grande régularité de l'activité EMG, on peut supposer que la distension utérine explique en partie ces différences. La plus longue durée des salves peut être aussi attribuée à l'ocytocine dont la présence a été mise en évidence dans le plasma fœtal au cours des 40 dernières heures de la gestation (Forsling et al., 1975) et dans le plasma maternel au cours de l'expulsion de l'agneau (Chard, 1972 ; Chard, 1974). D'ailleurs des salves comparables à celles recueillies au cours des dernières heures de la gestation sont enregistrées après une injection de 5 Ul d'ocytocine chez la brebis en œstrus.

II ressort aussi de cette étude que, dans nos conditions, on n'observe pas de différence entre l'évolution de l'activité utérine des brebis qui ont mis bas spontanément et celle des brebis traitées par la dexaméthasone. Après administration de ce composé, la diminution du taux de la progestérone et l'élévation du faux des œstrogènes plasmatiques sont comparables à celles qui se produisent normalement avant l'agnelage (Bosc, Fèvre, Terqui, communication personnelle). La modification de l'activité EMG de l'utérus qui survient le dernier jour de la gestation chez la brebis traitée comme chez la brebis témoin, semble donc être reliée de façon assez constante à ces variations hormonales plutôt qu'à l'injection de dexaméthasone. En effet, ce changement se produit à des intervalles variables selon les animaux après l'administration de ce corticostéroïde dont le taux est relativement élevé dans le plasma maternel au cours des $12 \mathrm{~h}$ suivant l'injection (Bosc et Terqui, 1976). On peut conclure que la dexaméthasone n'exerce pas par elle-même un effet stimulateur direct sur l'activité utérine lorsqu'elle est utilisée pour induire l'agnelage.

Accepté en juin 1976.

Remerciements. - Nous remercions le Professeur J.-P. Rousseau pour les conseils et les commentaires qu'il nous a prodigués au cours de ce travail. 


\section{Références}

BASSET J. M., OXBORROW T. J., SMITH I. D., THORBURN G. D., 1969. The concentration of progesterone in the peripheral plasma of the pregnant ewe. J. Endocr., 45, 449-457.

BOSC M. J., 1970. Etude de l'induction de la parturition chez la brebis par un corticostéroïde de synthèse, la fluoro-méthyl prednisolone. C. R. Acad. Sc., Paris, 270, D, 3127-3130.

BOSC M. J., 1972. Conséquence sur la parturition de l'hypophysectomie de la mère ou du foetus, chez la brebis traitée par la dexaméthasone C. R. Acad. Sc., Paris, 274, D, 93-96.

BOSC M. J., FEVRE J., 1974. Etude du mode d'action de la dexaméthasone utilisée pour induire l'agnelage chez la brebis. C. R. Acad. Sc., Paris, 278, D, 315-318.

BOSC M. J., FEVRE J., 1975. Evolution de la progestéronémie chez la brebis en fin de gestation après destruction de l'hypophyse foetale. Effet d'une administration de dexaméthasone. C. R. Acad. Sc., Paris, 281, D, 559-562.

BOSC M. J., TERQUI M., 1976. Etude du passage placentaire de la dexaméthasone après hypophysectomie du fœetus chez la Brebis. Ann. Biol. anim. Bioch. Biophys., à paraitre.

CHALLIS J. R. G., HARRISON F. A., HEAP R. B., HORTON E.W., POYSER N. L., 1972. A possible role of cestrogens in the stimulation of PG F2 $\alpha$ output at the time of parturition in a sheep. $J$. Reprod. Fert., 30, 485-488.

CHARD T., 1972. The posterior pituitary in human and animal parturition. J. Reprod. Fert., Suppl. 16, 121-138.

CHARD T., 1974. The role of fetal and maternal oxytocin in the onset of spontaneous labour. In Avortement ef parturition provoqués. 169-177. Colloque de la Société Nationale pour l'Etude de la Stérilité ef de la Fécondité. Paris, Masson et Cie éd.

CURRIE W. B., WONG M. S. F., COX R. I., THORBURN G. D., 1973. Spontaneous or dexamethasone-induced parturition in the sheep and goat : changes in plasma concentration of maternal prostaglandin F and fotal œstrogen sulphate. Mem. Soc. Endocr., 20, 95-118.

DROST M., HOLM L.W., 1968. Prolonged gestation in ewes after fœtal adrenalectomy. J. Endocr., 40, 293-296.

FORSLING M., JACK P. M. B., NATHANIELSZ P.W., 1975. Plasma oxytocin concentrations in the fotal sheep. Horm. Metab. Res., 7, 197.

HINDSON J. C., SCHOFIELD B. M., TURNER C. B., WOLFF H. S., 1965. Parturition in the sheep. J. Physiol. Lond., 181, 560-567.

HINDSON J. C.,WARD W. R., 1973. Myometrial studies in the pregnant sheep. In The endocrinology of pregnancy and parturition, experimental studies in sheep. 159-162. Symp. Cardiff, 1971. Alpha Omega Alpha publ. Cardiff.

LIGGINS G. C., KENNEDY P. C., HOLM L.W., 1967. Failure of initiation of parturition after electrocoagulation of the pituitary of the fœetal lamb. Am. J. Obsfet. Gynec, 98, 1080-1086.

LIGGINS G. C., 1968. Premature parturition after infusion of corticotrophin or cortisol into fotal lambs. J. Endocr., 42, 323-329.

LIGGINS G. C., FAIRCLOUGH R. J., GRIEVES S. A., KENDALL J. Z., KNOX B., 1973. The mechanism of initiation of parturition in the ewe. Rec. Progr. Horm. Res., 29, 111-159.

MOSSMAN R. G., CONRAD J. T., 1969. Oxytocic and modulation effects of water-soluble hydrocortisone and methyl-prednisolone upon in vifro contractions of myometrium. Am. J. Obsfef. Gynec., 105, 897-908.

NAAKTGEBOREN C., POOL C., VAN DER WEIJDEN G. C., TAVERNE M. A. M., SCHOOF A. G., KROON C. H., 1975. Elektrophysiologische Untersuchungen über die Uteruskontraktionen des Schafes während der Trächtigkeit und der Geburt. Z. Tierzüchłg. Züchtgsbiol, 92, 220-243. 
ROBERTSON H. A., SMEATON T. C., 1973. The concentration of unconjugated cestrone, cestradiol$17 \alpha$ and cestradiol-17 $\alpha$ in the maternal plasma of the pregnant ewe in relation to the initiation of parturition and lactation. J. Reprod. Fert. 35, 461-468.

ROUSSEAU J. P., PRUD'HOMME M. J., 1974. Etude électromyographique de la motricité de l'utérus chez la Brebis. Action des hormones. Ann. Biol. anim. Bioch. Biophys., 14, 67-85.

THORBURN G. D., NICOL D. H., BASSET J. M., SCHUTT D. A., COX R. 1., 1972. Parturition in the goat and sheep : changes in corticosteroïds, progesterone, œstrogens and prostaglandin $F$. J. Reprod. Ferf., suppl. 16, 61-84.

ZASZTOWT O.,WISNIEWSKI J., 1973. The influence of cortisol on uterine contractility in pregnancy and labor. 7e WId Congr. Obstet. Gynec. Moscou. Aoat 1973. Excerpto Medica, I. C. S. no 279, 29-30. 